\title{
Unspezifische Reize - ein klassisches Missverständnis
}

In Veröffentlichungen über Naturheilverfahren und Komplementärmedizin, aber auch über Balneologie und Physikalische Medizin, wird häufig von «unspezifischen Reizen» gesprochen. Sie werden zur Erklärung des bekannten Phänomens herangezogen, dass die Verfahren der natürlichen Therapie [1] häufig sehr unterschiedliche Wirkungen besitzen, die darüber hinaus noch sehr unterschiedliche Funktionen, Organe der Organsysteme bereffen können. So können dieselben Therapien z.B. in gleicher Weise bei Verdauungskrankheiten und Schlafstörungen oder funktionellen Kreislaufbeschwerden wirksam sein. Auch die Tatsache, dass unterschiedliche Therapieformen oft gleichartige Wirkungen besitzen, wird mit dem Theorem der «unspezifischen Reize» erklärt. Es beruht allerdings auf einem grundlegenden Missverständnis und entspricht nicht den Erkenntnissen der modernen Physiologie.

Die verwendeten therapeutischen Reize sind durchweg hochspezifisch [2] und physikalisch oder chemisch eindeutig definiert. So sind z.B. die Reize Wärme und Kälte als Abweichung vom Thermoneutralpunkt, mechanische Stimuli wie Druck oder Vibration oder die Parameter der Bewegung physikalisch klar gekennzeichnet und voneinander grundverschieden. Gleiches gilt für chemische Wirkstoffe wie Kohlenstoffdioxid, Elektrolyte oder Pflanzeninhaltsstoffe. Alle diese Einwirkungen treffen im Organismus auf hochspezifische Rezeptorsysteme. So ist seit langem allgemein bekannt, dass schon die Temperaturreize Wärme und Kälte eigene sich unterscheidende Rezeptoren erregen und darüber hinaus z.T. gegensätzliche Wirkungen hervorrufen (z.B. Vasokonstriktion oder Vasodilatation). Gleiches gilt für die verschiedenen mechanischen Einwirkungen, für die eine Reihe verschiedener Rezeptoren zur Verfügung stehen (Druckrezeptoren, Spannungsrezeptoren usw.). Auch die chemischen Wirkungen in der Balneologie oder Phytotherapie sind hochspezifisch und genau definiert. So ist der Angriffspunkt von $\mathrm{CO}_{2}$ am Gefässsystem gut aufgeklärt, und dessen Effekte sind spezifisch. Auch bei den inner- lichen Wirkungen von Pflanzeninhaltsstoffen liegen solche spezifischen Rezeptorwirkungen zugrunde, was allerdings nicht ausschliesst, dass einzelne Inhaltsstoffe auch mehrere Wirkungen haben und verschiedene Stoffe auch die gleichen Rezeptoren reizen können. Es besteht also kein Zweifel, dass die Primärwirkungen bzw. -reaktionen auf die verschiedenen therapeutischen Reize ebenfalls hochspezifisch sind.

Wie kommt es nun zu dem einleitend genannten Phänomen der unspezifischen Wirkungen in verschiedenen Organ- und Funktionssystemen und den gleichartigen Wirkungen unterschiedlicher Therapien? Dies ist in den adaptiven Langzeitwirkungen der wiederholten Reizanwendung begründet. So führt jeder Stimulus, der auf den Organismus einwirkt und die individuell gewohnte Regulationskapazität überschreitet, zu adaptiven Antworten, deren Ziel es letztendlich ist, die Regulationskapazität auszuweiten, und zwar entweder durch eine Zunahme der Reaktionsökonomie («funktionelle Adaptation») oder durch Ausweitung der Reaktionskapazität («trophische und/oder plastische Adaptation») [1]. Voraussetzung für solche adaptiven Antworten ist in der Regel eine wiederholte serielle Reizeinwirkung. Häufig zu beobachten ist auch, dass die primären Reizwirkungen und das langfristige Adaptationsergebnis unterschiedlich oder sogar gegensätzlich sind. Bei diesen adaptiven Reaktionen werden häufig autonome bzw. vegetative Hintergrundreaktionen mit einbezogen, deren Reaktionsanteile tatsächlich unspezifisch sind und die auch gleichartige Reaktionen in unterschiedlichen Systemen bewirken können. So kann z.B. eine Anwendung, die primär den (systolischen) Blutdruck steigert, wie z.B. die Ausdauerbelastung des Herz-Kreislauf-Systems, genauso wie eine, die den Blutdruck primär senkt (z.B. $\mathrm{CO}_{2}$-Vollbäder) langfristig zu gleichartigen Veränderungen des Blutdruckverhaltens führen. Bei diesen langfristigen Veränderungen, die der funktionellen Adaptation zuzurechnen sind, sind nicht die primären Reizreaktionen massgebend, vielmehr werden die reaktiven Ant-

\begin{tabular}{ll}
\hline KARGER & @ 2003 S. Karger GmbH, Freiburg \\
$\begin{array}{l}\text { Fax +49 7614520714 } \\
\begin{array}{l}\text { E-mail Information@Karger.de } \\
\text { www.karger.com }\end{array}\end{array}$ & $\begin{array}{l}\text { Accessible online at: } \\
\text { www.karger.com/fkm }\end{array}$
\end{tabular}

Univ.-Prof. Dr. med. Christoph Gutenbrunner Institut für Balneologie und Medizinische Klimatologie Medizinische Hochschule Hannover

Carl-Neuberg-Str. 1

D-30625 Hannover

E-mail gutenbrunner.christoph@mh-hannover.de 
worten vom Funktionszustand und der Reaktionsfähigkeit des behandelten Organismus geprägt. Bezüglich der Reaktion auf den Reiz ist hier die Bezeichnung «unspezifisch» also tatsächlich gerechtfertigt.

Aber auch bei den adaptiven Reaktionen kann nur ein Teil als unspezifisch aufgefasst werden. Im Wesentlichen unspezifisch sind tatsächlich die funktionellen Adaptationen. Sie sind über das Hypophysen-Nebennierenrinden-System gesteuert und haben daher im Sinne positiver Kreuzadaptationen auch Wirkungen in sehr unterschiedlichen Funktionssystemen. Anders ist es allerdings bei den in der Rehabilitation besonders wichtigen trophischen und plastischen Adaptationen. Als Wachstumsprozesse sind sie in ihren Effekten in der Regel spezifisch, z.B. das Wachstum von Myofibrillen beim Muskeltraining oder die Erythropoese bei Sauerstoffmangelreizen. Sie werden von spezifisch wirkenden Hormonsystemen gesteuert (STH, Erythropoetin u.a.). Sie bewirken keine positiven Kreuzadaptationen, sondern können sogar zu Funktionsverlusten in anderen Organen oder Systemen führen. Besonders deutlich wird dies an den bekannten unterschied- lichen Effekten des isometrischen oder dynamischen Muskeltrainings. Eine genauere Differenzierung zwischen spezifischen und unspezifischen Reaktionsanteilen bei neuralen (Koordination, Neuroplastizität) und kortikalen Adaptationen (Lernprozesse) steht allerdings noch aus. $\mathrm{Zu}$ beachten ist aber, dass gleichartige Reize durchaus Adaptationen unterschiedlicher Art auslösen und so gleichsam spezifische wie auch unspezifische adaptive Reaktionen hervorrufen können. Insgesamt gilt also die Regel: Alle Reize sind spezifisch, die von ihnen ausgelösten Reaktionen hingegen besitzen neben spezifischen auch unspezifische Anteile.

C. Gutenbrunner, Hannover

\section{Literatur}

1 Hildebrandt G: Therapeutische Physiologie; in Gutenbrunner C, Hildebrandt G (Hrsg.): Handbuch der Balneologie und medizinischen Klimatologie. Berlin, Springer, 1998, pp 5-84.

2 Gutenbrunner C: Therapieprinzipien; in Gutenbrunner C, Weimann G (Hrsg.): Krankengymnastische Methoden und Konzepte. Berlin, Springer, 2003, pp 9-24. 